\title{
The Role Of Culture In Diagnosing Smear Negative Tuberculosis In HIV Seropositive Patients.
}

\author{
Umamaheshwari $\mathrm{S}^{1}$, Sumana $\mathrm{MN}^{2}$ \\ ${ }^{I}$ Research Scholar,Department of Microbiology, JSS Medical College, JSS University, India. \\ ${ }^{2}$ Professor of Microbiology, Department of Microbiology, JSS Medical College, JSS University, India.
}

\begin{abstract}
Sputum microscopy, the most commonly practiced tuberculosis (TB) diagnostic test is less sensitive in immunocompromised patients due to low bacillary load.The study was carried out in a tertiary care general hospital during 2010-12 in Mysore, Karnataka, to diagnose smear negative TB in HIV patients by culture technique. Of416 HIV patients, 162 patients with features of pulmonary/extrapulmonary TB but smear negative were included in the study.Sputum, stool, blood and other relavent clinical samplese except blood were processed as per standard protocol. The blood collected with sodium citrate was processed by lysiscentrifugation. All samples were inoculated onto Lowstein Jensen slants and incubated at $37^{\circ} \mathrm{c}$ for 6 to 8 weeks. Of 162 HIV patients, 67(41\%) were found to have TB. Extrapulmonary TB (25\%) was more common than pulmonary TB (13\%). Mycobacteria were recovered in 76 samples (26 sputum, 12 stool, 18 blood, 7 pleural fluid, 1 CSF, 9 FNAC, 1 ascitic fluid, 1pus , 1 ear discharge). M.tuberculosis (95\%) was the predominant species isolated followed by M.avium complex (5\%).Most HIV patients with TB are left undiagnosed for reasons of no advanced/high cost techniques in resource constrained settings. Thus culture could be used as a tool in diagnosing smear negative TB.
\end{abstract}

Keywords: Culture, HIV, Smear negative, Tuberculosis.

\section{Introduction}

Human Immunodeficiency Virus (HIV) infection and Tuberculosis (TB) increase the rate of disease progression of each other, thereby diminishing the survival time of the patients. Among HIV patients, TB is the most common opportunistic infection that can occur at any stage of HIV infection. The clinical presentation is influenced by the degree of immunosuppression. TB is often atypical in presentation, frequently causing extrapulmonary disease in HIV patients. Diagnosing smear negative TB in resource constrained settings is a challenging task. Sputum microscopy, the most commonly practiced TB diagnostic test in many low income countries detects $<50 \%$ of TB cases in immunocompromised patients. The sensitivity of this test is less due to absence or reduction in cavitation resulting in low bacillary load in HIV positive patients. HIV infection even diminishes the reliability of chest radiograph in the diagnosis of pulmonary tuberculosis as the disease commonly presents with atypical pattern. ${ }^{[1,2,3]}$ To accelerate the development of TB diagnostics for people with HIV, World Health Organization (WHO) released guidelines in 2006 - 2007 stating culture of sputum (smear negative) and other clinical samples with a clinical suspicion of TB. ${ }^{[4,5]}$

Culture of clinical specimen is more sensitive than smear microscopy because 10-100 viable organisms will result in a positive culture whereas 5000-10 000 acid-fast bacilli (AFB) per $\mathrm{ml}$ are required for detection by smear ${ }^{[6]}$ Though BACTEC and other systems are providing the most rapid methods for mycobacterial detection, isolates obtained from cultures can be used for species identification, drug susceptibility and molecular epidemiology ${ }^{[7-9]}$ Christie and Callihan suggests that the techniques used to diagnose mycobacterial disease should match with the resources available in the laboratory and the incidence of the disease. ${ }^{[10]}$ Also, the incidence of mycobacteremia is dramatically raised in patients with AIDS. Culture being a gold standard as recommended by WHO, various clinical samples collected from the site of pathology in smear negative patients were cultured to recover Mycobacteria and to assess the usefulness.

\subsection{Study Setting:}

\section{Materials And Methods}

The study was conducted at a tertiary care general hospital located in the center of Mysore city, Karnataka, India during Aug 2010 to Dec 2012.It caters to patients coming from Mysore, Chamarajnagar, Mandya, Hassan, Hegaddevankote and Coorg districts.

\subsection{Sample Population:}

Totally 416 patients were found to be HIV seropositive during the study period and 162 patients with signs and symptoms suggestive of pulmonary/extra pulmonary tuberculosis butsmear negative for AFB 
constituted the study population. Ethical approval was obtained from the Institutional Ethics Committee, and accordingly informed written consent was obtained from all patients.

\subsection{Selection Criteria:}

Inclusion Criteria: HIV reactive patients with any of the following features were included as study subjects; a history of prolonged fever, weight loss, cough for more than 2 weeks (sputum smear negative), radiological evidence suggestive of $\mathrm{TB}$, pleural effusion, diarrhoea persisting for $>1$ month, pain abdomen/ascites/ lymphadenopathy or any other features suggestive of TB.

Exclusion Criteria: HIV seronegative patients, HIVseropositive patients with no features of TB or smear positive for AFB and patients with diarrhoea due to parasitic infestation.

\subsection{Sample collection and Processing:}

A detailedhistory of each patient was entered in the proforma. Each patient was instructed to provide sputum (if productive) and stool at early morning for AFB smear and culture. Also, blood and other clinical specimens depending on the site of pathology were collected by aseptic methods.

Approximately $1 \mathrm{gm}$ of stool sample was emulsified in $5 \mathrm{ml}$ of Middlebrook 7H9 broth.The stool and sputum specimens were decontaminated by adding equal amounts of $4 \%$ sodium hydroxide $(\mathrm{NaOH})$ and incubated for $20 \mathrm{~min}$ at $37^{\circ} \mathrm{C}$.All specimens (body fluids if any) except blood were concentrated by centrifugation for $30 \mathrm{~min}$ at $3000 \mathrm{~g}$. Smears of the centrifuged deposit were prepared and stained by Ziehl Neelson (ZN) technique for detection of AFB and inoculated onto Lowstein Jensen (LJ) slant for culture.

Lysis centrifugation technique was followed to process blood samples. Approximately $5 \mathrm{ml}$ of venous blood collected was transferred immediately into $15 \mathrm{ml}$ sterile centrifuge tube with $300 \mu \mathrm{l}$ of $3.8 \%$ sodium citrate anticoagulant. The tubes were centrifuged at $3000 \mathrm{~g}$ for $10 \mathrm{~min}$ and serum was discarded. The buffycoat was aseptically transferred into Wintrobe tube using a lumbar puncture needle (LP) and centrifuged at $3000 \mathrm{~g}$ for 30 min. Concentrated buffycoat was transferred using LP needle into a sterile ependoff tube containing $50 \mu \mathrm{l}$ of $0.1 \%$ saponin. To enhance the lysis of cells, the mixture was vortexed with coarse glass beads for $10-12$ min. From this two smears were prepared and stained by Leishman and $\mathrm{ZN}$ stains for the confirmation of cell lysis and presence of AFB respectively. The sample was then inoculated onto LJ medium.

All the LJ slants were incubated at $37^{\circ} \mathrm{C}$ for 6 to 8 weeks and were examined for growth twice a week. Any growth observed was confirmed to be Mycobacteria by ZN staining of the culture smear.

\section{Results}

A total of $162 \mathrm{~TB}$ suspected patients out of $416 \mathrm{HIV}$ positive patients were included in the study. The age of these patients ranged from 18 to $97 \mathrm{yrs}$. The majority were in the age group of $31-40 \mathrm{yrs}$ (70, $43 \%$ Table1). Of 162 patients $124(77 \%)$ were males and $38(23 \%)$ were females.

Totally 76 Mycobacterial isolates were recovered out of 443 clinical samples collected from 162 patients (26 sputum, 12 stool, 18 blood samples, 7 pleural fluid (PF), 1 cerebrospinal fluid (CSF), 9 fine needle aspiration cytology (FNAC) samples, 1 ascitic fluid (AF), 1pus and 1 ear discharge - Table 2). Out of 162 patients, 67(41\%) patients yielded growth of Mycobacteria. In some patients, Mycobacteria were isolated from more than one sample (both sputum and stool in 4 patients, stool and blood in 1 patient, sputum and blood in 1 patient, $\mathrm{AF}$ and blood in 1 patient, $\mathrm{PF}$ and blood in 1 patient and in FNAC and blood in 1 patient) and in most patients from a single sample (21 from sputum, 7 from stool, 13 from blood, 8 from FNAC, 6 from PF, 1 from pus, 1 from CSF and 1 from ear discharge).

Of the 76 Mycobacterial isolates 69 (95\%) were Mycobacterium tuberculosis (MTB) and 7(5\%) were Mycobacterium avium. MTB was recovered from 24 sputum, 16 blood, 10 stool, 9 FNAC, 7 pleural fluid, 1 CSF, 1 Ascitic fluid, 1 pus and M.avium from 2 sputum, 2 stool, 2 blood and 1 ear discharge samples (Table 2).

Pulmonary TB was detected in 21(13\%) patients and extrapulmonary TB in $41(25 \%)$ patients and 5 (3\%) patients had both.Chi Square Test applied to each clinical sample which yielded growth of Mycobacteria showed that pleural fluid (0.394) and FNAC(1.000) were more significant compared to rest of the samples

TABLE 1: Age wise distribution of cases.

\begin{tabular}{|l|c|c|}
\hline \multicolumn{1}{|c|}{ Age } & No. of cases & Percentage \\
\hline $0-20$ yrs & 1 & 1 \\
\hline $21-30$ yrs & 26 & 16 \\
\hline $31-40$ yrs & 70 & 43 \\
\hline $41-50$ yrs & 33 & 20 \\
\hline $51-60$ yrs & 24 & 15 \\
\hline 60 yrs above & 8 & 5 \\
\hline Total & 162 & 100 \\
\hline
\end{tabular}


TABLE 2: Recovery of Mycobacterial isolates from different clinical samples

$\begin{array}{lccrr}\text { Specimen(s) } & \text { Sample No. } & \text { Culture positive(\%) } & \text { M.tuberculosisM.avium } \\ \text { Blood } & 162 & 18(11) & 16 & 2 \\ \text { Bone } & 3 & 0(0) & 0 & 0 \\ \text { Cerebrospinal fluid } & 20 & 1(5) & 1 & 0 \\ \text { FNAC } & 22 & 9(41) & 9 & 0 \\ \begin{array}{l}\text { Others (pus, ear discharge, } \\ \text { skin lesions, ascitic fluid) }\end{array} & 33 & 3(9) & 2 & 1 \\ \text { Pleural fluid } & 14 & 7(50) & 7 & 0 \\ \text { Sputum } & 74 & 26(35) & 24 & 2 \\ \text { Stool } & 115 & 12(10) & 10 & 2 \\ \text { Total } & \mathbf{4 4 3} & \mathbf{7 6} & \mathbf{6 9} & \mathbf{7}\end{array}$

\section{Discussion}

As per WHO, 5 million people are living with HIV in South, South- East and East Asia. ${ }^{[1]}$ People with HIV have 20-30 times higher lifetime risk of developing active TB, compared to people without HIV. ${ }^{[12]}$ In 2010, people living with HIV accounted for about $13 \%$ of tuberculosis cases worldwide, and about 3,60,000 died from HIV-related tuberculosis. ${ }^{[13]}$ Although patients with sputum smear-negative TB are less infectious, they are more likely to die during or before diagnosis than patients with smear-positive TB and contribute to TB transmission. ${ }^{[14,15]}$ A follow up study at Malawai reported that smear negative pulmonary TB patients had higher risk of death than smear positive TB with a hazard ratio of 2.2. ${ }^{[16]}$ Autopsy studies of HIV positive patients identified TB as a cause of death in $14-54 \%$ of adults or adolescents. ${ }^{[17-19]}$.

Mycobacteremia has now become common in patients with advanced HIV infection and active TB. ${ }^{[20,}$ ${ }^{21]}$ Screening of TB among HIV patients with symptoms of fever, cough, weight loss and chest radiography would detect only $25 \%$ of HIV-TB co-infected patients. ${ }^{[22]}$ The culture technique employed in this study detected TB in 67 (41\%) out of 162 HIV seropositive, smear negative patients. Out of the 443 clinical samples collected, 76 samples yielded growth of Mycobacteria. Our HIV-TB co-infection rate of $41 \%$ concurs with the studies of Zambia -43\%, ${ }^{[23]}$ Pune - 45.3\% ${ }^{[24]}$ Vadodara,Gujarat- 49.2\%, ${ }^{[25]}$ South African- 49\% ${ }^{[26]}$ Southern California $43.2 \%{ }^{[27]}$ and Benin, Nigeria $-33.9 \%{ }^{[28]}$ Wide disparity was observed in a Tanzanian study which showed low co-infection rate of $5.3 \% .{ }^{[2]}$ Hence the early TB detection among HIV patients is vital.

In the present study, number of extrapulmonary cases $(41-25 \%)$ were more than pulmonary cases (21$13 \%)$ which concur with studies of Shimla (25.28\% pulmonary TB and $57.47 \%$ extrapulmonary $\mathrm{TB})^{[29]}$ and Vadodhara, Gujarat $(55 \% \text { pulmonary and } 68 \% \text { extrapulmonary } \mathrm{TB})^{[25]}$ but differs from Benin, Nigeria $(88 \%$ pulmonary and $12 \%$ extrapulmonary TB) ${ }^{[28]}$ and Zambian sudies (40\% pulmonary , 34\% extrapulmonary). ${ }^{[30]}$

In most of the western countries Mycobacterium avium complex (MAC) is the leading cause of TB in HIV infected patients whereas in India, M.tuberculosis is the commonest cause ${ }^{[20]}$ In this study MTB $(69-95 \%)$ was the predominant species isolated followed by M. avium (7-5\%) which matches with the study of Buenos Aires wherein MTB was isolated in $223(92.9 \%)$ andMAC in 14 patients (5.8\%). The other two species identified in their study were M. Kansasiand M. bovis. ${ }^{[31]}$ Our study differs from studies of Praharaj et al of Pune ${ }^{[24]}$ who reports only MTB with no atypical Mycobacteria and study of Murcia-Aranguren et al of Bogota, in which M.avium was the predominant species $13(4.2 \%)$ and MTB was only $4(1.4 \%) .{ }^{[32]}$

In this study blood culture was found to be positive in $18(11 \%)$ out of 162 samples and 13 patients were found to have TB only by blood culture. Of 18 isolates, MTB was isolated in 16 and MAC from 2. The present study concurs with Truffot-Pernot Cet al studies where 61 (10.8\%) samples taken from 19 patients $(11.5 \%)$ were positive by blood culture and $\mathrm{M}$. avium intracellulare was the most frequently isolated species. ${ }^{[33]}$ Studies of M Di Lonardoet al of Buenos Aires differs from this study wherein blood culture was positive in $21.2 \%$ which is almost double while Patama Monkongdee et al of Thailand study showed only $2 \%$ positivity (16 out of 1051) . $^{[31,34]}$

In South African study ${ }^{[26]} 49 \%$ of patients who were smear negative showed culture positivity in sputum which slightly differs from our study that shows lower percentage i.e., 26 (35\%) out of 74 sputum samples.

Stool culture was positive in 12 (10\%) out of 115 samples in the present study which slightly differs from Thailand study where only $61(6 \%)$ stool showed culture positivity out of 1052 samples. ${ }^{[34]}$ Evidence of MTB in the stool may be attributed to gastrointestinal TB or to sputum swallowed by persons with pulmonary $\mathrm{TB}$; in patients with chronic diarrhea, yield in stool culture may be particularly high ${ }^{[35-37]}$ Patama Monkongdee et al found it a problem to recommend stool culture as an adjunct to diagnose TB because of difficulty in decontamination. ${ }^{[34]}$ As per our findings though $10 \%$ stool were culture positive, very minimal $(<1 \%)$ contamination rate was observed in the technique we followed. 
In our study 7 pleural fluid samples showed growth out of 14 samples which differs from Elliot and Halwiindi et al study which shows pleural fluid culture positivity in $12(26 \%)$ out of 46 patients. ${ }^{[30]}$

In this study FNAC showed culture positive in 9 (41\%) out of 22 samples which concurs with Thailand study (34 out of 82 lymphnode aspirates-42\%). ${ }^{[34]}$

More rapid and more sensitive techniques have replaced traditional methods of direct examination and culturing for diagnosing mycobacterial infections. Among the most recent methods are Isolator blood culture, radiometric detection, hybridization and amplification, each with their own advantages and disadvantages. ${ }^{[38]} \mathrm{Also}$ these are costlier compared to conventional culture technique.

Limitation of our study was that a small proportion of TB suspected patients were recognized since it was hospital based and the patients included were those who were seriously ill and hospitalized. Thus, we might have missed patients who were out patient/not seriously ill/not opted to attend the hospital for care.

\section{Conclusion}

Diagnosis of tuberculosis in PLWH is extremely important in order to plan a therapeutic conduct. The occurrence of TB in HIV accounted for $41 \%$ and the extrapulmonary presentation of TB were high in this study. India being a high prevalent area in HIV and TB diseases, physicians should have a high clinical suspicion of TB in HIV patients and all HIV individuals should be screened for TB. If patients are diagnosed at an early stage of disease, the mortality and transmission rate can be reduced as TB is treatable. Culturing sputum and other clinical samples from suspected TB in HIV seropositive cases should be encouraged as part of routine procedure in diagnosing TB. Many patients are left undiagnosed for TB for reasons of no advanced /high cost techniques available. In such situations, existing facilities like culture can be completely utilized. Culture being cost effective, sensitive and a gold standard, can be routinely used in the laboratory as a tool in diagnosing smear negative TB cases.

\section{Acknowledgement}

Our sincere thanks to Indian Council of Medical Research (ICMR-IRIS No.5/8/5/8/2008-ECD-I), New Delhi for financial assistance in carrying out this study.We also thank Mr B Mahadevaswamy, Dr K Jayashree, Dr K Anurada, Dr B Sumangala for their valuable inputs for our study.

\section{References:}

[1]. Source: HIV and Hepatitis C Annual Update. Management of Tuberculosis in HIV-Infected Patients. 2012. [cited 2012 Dec 31]. Available from

http://www.clinicaloptions.com/HIV/Annual\%20Updates/2012\%20Annual\%20Update/Modules/TB_HIV_Management.aspx

[2]. Elston JW, Thaker HK, Co-infection with human immunodeficiency virus and tuberculosis,Indian J DermatolVenereolLeprol74(3),2008,194-199.

[3]. Cattamanchi A, Davis JL, Worodria W, YOO S, Matovu J, Kiidha J, et al,Poor performance of universal sample processing method for diagnosis of pulmonary tuberculosis by smear microscopy and culture in Uganda,J ClinMicrobiol,46(10),2008,3325-9.

[4]. World Health Organization. Geneva: Improving the diagnosis and treatment of smear-negative pulmonary and extrapulmonary tuberculosis among adults and adolescents: recommendations for HIV-prevalent and resource-constrained settings. 2006.

[5]. WHO Strategic and Technical Advisory Group for Tuberculosis. Report on conclusions and recommendations, 11-13 June, 2007, WHO Headquarters, Geneva, Switzerland. [cited 2012 Dec 31]. Available from: http://www.who.int/tb/events/stag_report_2007.pdf.

[6]. Rouillon A, Perdrizet S, Parrot R, Transmission of tubercle bacilli: The effects of chemotherapy, Tubercle, 57(4), 1976,275-299.

[7]. Heifets LB, Cangelosi GA, Drug susceptibility testing of Mycobacterium tuberculosis: a neglected problem at the turn of the century, Int J Tuberc Lung Dis,3(7),1999,564-581.

[8]. Drobniewski FA, Caws M, Gibson A, Young D, Modern laboratory diagnosis of tuberculosis, Lancet Infect Dis,3(3),2003,141-147.

[9]. Kiehn TE, Cammarata R, Laboratory diagnosis of mycobacterial infections in patients with acquired immunodeficiency syndrome, $J$ ClinMicrobiol,24(5),1986,708-711.

[10]. Christie JD, Callihan DR, The laboratory diagnosis of mycobacterial diseases. Challenges and common sense, Clin Lab Med, 15(2),1995,279-306.

[11]. Global aids epidemic. UNAIDS report. 2012.

[12]. Time to act save a million lives by 2015.Geneva, Stop TB Partnership, Joint Untitled Nations Programme on HIV/AIDS, World Health Organization.2011.

[13]. Global Tuberculosis Control, Geneva. WHO report 2011- [cited 2012 Dec 31]. Available from http://www.who.int/tb/publications/global_report/gtbr12_main.pdf.

[14]. Tostmann A, Kik SV, Kalisvaart NA, Sebek MM, Verver S, Boeree MJ, et al, Tuberculosis transmission by patients with smearnegative pulmonary tuberculosis in a large cohort in the Netherlands, Clin Infect Dis, 47(9),2008,1135-1142.

[15]. Getahun H, Harrington M, O'Brien R, Nunn P,Diagnosis of smear-negative pulmonary tuberculosis in people with HIV infection or AIDS in resource- constrained settings: informing urgent policy changes,Lancet,369(9578),2007,2042-2049.

[16]. Kang'ombe CT, Harries AD, Ito K, Clark T, Nyirenda TE, Aldis W, et al, Long-term outcome in patients registered with tuberculosis in Zomba, Malawai: mortality at 7 years according to initial HIV status and type of TB,Int J Tuberc Lung Dis,8(7),2004,829-836.

[17]. Ansari NA, Kombe AH, Kenyon TA, Hone NM, Tappero JW, Nyirenda ST, et al, Pathology and causes of death in a group of 128 predominantly HIV- positive patients in Botswana,1997-1998,Int J Tuberc Lung Dis,6(1),2002,55-63.

[18]. Rana FS, Hawken MP, Mwachari C, Bhatt SM, Abdullah F, Ng'ang'a LW, et al, Autopsy study of HIV-1-positive and HIV-1 negative adult medical patients in Nairobi, Kenya, J Acquir Immune DeficSyndr, 24(1), 2000, 23-29.

[19]. d'ArminioMonforte A, Vago L, Gori A, Antinori S, Franzetti F, Antonacci CM, et al, Clinical diagnosis of mycobacterial diseases versus autopsy findings in 350 patients with AIDS, Eur J ClinMicrobiol Infect Dis, 15(6),1996,453-458. 
[20]. McDonald LC, Archibald LK, Rheanpumikankit S, Tansuphaswadikul S, Eampokalap B, Nwanyanawu O, et al, Unrecognised Mycobacterium tuberculosis bacteraemia among hospital inpatients in less developed countries,Lancet,354(9185),1999,1159-1163.

[21]. Bouza E, Diaz- Lopez MD, Moreno S, Bernaldo de Quiros JC, Vicentre T, Berenguer J, Mycobacterium tuberculosis bacteremia in patients with and without human immunodeficiency virus infection, Arch Intern Med,153(4),1993,496-500.

[22]. Ngowi BJ, Mfinanga SG, Bruun JN, Morkve O, Pulmonary tuberculosis among people living with HIV/AIDS attending care and treatment in rural northern Tanzania, BMC Public Health, 8,2008,341.

[23]. Elliott AM, Namaambo K, Allen BW, Luo N, Hayes RJ, Pobee JO, et al, Negative sputum smear results in HIV-positive patients with pulmonary tuberculosis in Lusaka, Zambia,Tubercle Lung Dis,74(3),1993,191-194.

[24]. Praharaj AK, Kalghatgi AT, Varghese SJ, Nagendra A, Incidence and drug susceptibility pattern of mycobacterium tuberculosis in HIV infected Patients,Medical Journal Armed Forces India,60(2),2004,134-136. [cited 2012 Jan 28]. Available from http://www.sciencedirect.com/science/article/pii/S0377123704801035

[25]. GhiyaR,Naik E,CasanasB,Izurieta R, MarfatiaY,Clinico-epidemiological profile of HIV/TB coinfected patients in Vadodara, Gujarat,Indian J Sex Transm Dis,30(1), 2009,10-15.

[26]. Coetzee D, Hilderbrand K, Goemaere E, MatthysF,Boelaert M, Integrating tuberculosis and HIV care in the primary care setting in South Africa, Trop Med Int Health,9(6), 2004,A11-15.

[27]. Rodwell TC, Barnes RF, Moore M, Strathdee SA, Raich A, Moser KS, et al, HIV-tuberculosis coinfection in Southern California: evaluating disparities in disease burden,Am J of Public Health,100(1), 2010.

[28]. Affusim CC, Kesieme E, Abah VO, The Pattern of Presentation and Prevalence of Tuberculosis in HIV-Seropositive Patients Seen at Benin City, Nigeria,Pulmonology 2012. [cited 2012 Nov 28]. Available from http://www.hindawi.com/isrn/pulmonology/2012/326572/

[29]. Jaryal A, Raina R, Sarkar M, Sharma A,Manifestation of tuberculosis in HIV/AIDS patients and its relationship with CD4 count,Lung India28(4), 2011,263-266.

[30]. Elliott AM, Halwiindi B, Hayes RJ, Luo N, Tembo G, Machiels L, et al,.The impact of human immunodeficiency virus on presentation and diagnosis of tuberculosis in a cohort study in Zambia, J Tropl Med Hyg,96(1),1993,1-11.

[31]. Di Lonardo M, Isola NC, Ambroggi M, Rybko A, Poggi S. Mycobacteria in HIV-infected patients in Buenos Aires, Tuber Lung Dis76(3),1995,185-189.

[32]. Murcia-Aranguren MI, Gómez-Marin JE, Alvarado FS, Bustillo JG, de Mendivelson E, Gómez B, et al. Frequency of tuberculous and non-tuberculous mycobacteria in HIV infected patients from Bogota, Colombia. BMC Infectious Diseases 2001;1:21.

[33]. Truffot-Pernot C, Lecoeur, HF, MauryL,DautzenbergB,Grosset J, Results of blood cultures for detection of mycobacteria in AIDS patients,Tubercle,70(3),1989,187-191

[34]. Monkongdee P, McCarthy KD, Cain KP, Tasaneeyapan T, Nguyen HD, Nguyen TN, et a, Yield of acid-fast smear and mycobacterial culture for tuberculosis for diagnosis in people with human immunodeficiency virus, Am J RespirCrit Care Med, 180(9),2009,903-908.

[35]. Allen BW. Isolation of Mycobacterium tuberculosis from faeces, Med Lab Sci46(2),1989,101-106.

[36]. Manatsathit S, Tansupasawasdikul S, Wanachiwanawin D, Setawarin S, Suwanagool P, Prakasvejakit S, et a, Cause of chrohonicdiarrhea in patients with AIDS in Thailand: a prospective clinical and microbiological study, $J$ Gastroenterol,31(4),1996,533-537.

[37]. Kramer F, Modilevsky T, Waliany AR, Leedom JM, Barnes PF, Delayed diagnosis of tuberculosis in patients with human immunodeficiency virus infection, Am J Med,89(4),1990,451-456.

[38]. Pierre-AudigierC,Recent biological techniques for the diagnosis of mycobacterial infections, Presse Med, 23(14), 1994,665-670. 